\title{
Haemodynamic and anaesthetic management of patients undergoing endovascular therapy for cerebral vasospasm
}

\author{
Audrey Tan, Suresh Tharmaradinam, Ramamani Mariappan, Pirjo Manninen, \\ Lakshmi Venkatraghavan
}

\begin{abstract}
Background: Cerebral vasospasm is a common and devastating complication after a subarachnoid haemorrhage (SAH). Current guidelines for treatment recommend hypertension with euvolaemia. Endovascular therapy with cerebral angioplasty and possible administration of intra-arterial vasodilators is indicated in patients who fail medical treatment. The objective of our study was to review the haemodynamic management and anaesthetic care of patients undergoing endovascular therapy for cerebral vasospasm in our institution. Materials and Methods: The medical records of all patients who underwent endovascular therapy for cerebral vasospasm between, April 2006 and September 2012 , were reviewed retrospectively. Patients with clinical vasospasm were treated initially by inducing hypertension to systolic pressures of I 40 to $170 \mathrm{mmHg}$; Endovascular treatment was performed, if there was no clinical improvement. Data was collected on blood pressure measurements, anaesthetic management, duration and complications of hypertensive therapy and outcome. The differences in the pre- and post-angioplasty systolic blood pressure were statistically analysed. Results: A total of 45 patients had 47 endovascular interventions, with balloon angioplasty for proximal vessel spasm and 16 (34\%) patients had additional intra-arterial injection of a vasodilator agent. Onset of vasospasm was 7 days (range 2-I 5 days) after SAH.Vasospasm was usually seen in multiple vessels in the same patient regardless of the site of ruptured aneurysm and was present unilaterally in $80 \%$ of the patients. All patients had a general anaesthesia for the procedure. Prior to endovascular treatment $68.9 \%$ patients required vasopressors, but post angioplasty $93.3 \%$ required them. Norepinephrine was the most commonly used (66.2\%). Angioplasty was successful in reversing the cerebral vasospasm as assessed by angiography in all patients with no intra-procedure complications. Overall $80 \%$ of patients were discharged from hospital to home or to a rehabilitation centre. Conclusion: Cerebral vasospasm affects multiple vessels in the same patient. Despite endovascular therapy being a successful intervention for proximal vessel spasm, most patients still required induced hypertension with even higher levels post angioplasty compared to pre angioplasty.
\end{abstract}

Key words: Anaesthesia, cerebral vasospasm, haemodynamic management

\begin{tabular}{|l|l|}
\hline \multicolumn{2}{|c|}{ Access this article online } \\
\hline Quick Response Code: & Website: \\
\hline & www.jnaccjournal.org \\
\hline & \\
\hline
\end{tabular}

\section{INTRODUCTION}

Cerebral vasospasm is one of the most common and devastating complications after an aneurysmal subarachnoid haemorrhage (SAH). Delayed cerebral ischaemia and infarction are the consequences of vasospasm that result in significant morbidity and mortality leading to a large social and economic burden. ${ }^{[1]}$

Department of Anesthesia, Toronto Western Hospital, University Health Network, University of Toronto, Toronto, Ontario, Canada

Address for correspondence:

Dr. Lashmi Venkatraghavan, Department of Anesthesia, Toronto Western Hospital, University Heath Network, University of Toronto. 399 Bathurst Street, Toronto, Ontario, M5T 2S8, Canada. E-mail: Lashmi.Venkatraghavan@uhn.ca 
Cerebral vasospasm develops in nearly half of all patients, who survive the first 24 hours and usually occurs 7-10 days after theSAH. ${ }^{[2]}$ The exact mechanism of vasospasm is still unclear. Traditionally, the management of vasospasm has been providing medical treatment with triple-H therapy, which aims for hypervolaemia, hypertension, and haemodilution to improve cerebral perfusion beyond the constricted arteries. The triple-H theory was introduced in 1982, but the current guidelines recommend only hypertension with euvolaemia. ${ }^{[3,4]}$ Currently, there is no consensus on the degree of hypertension needed with target blood pressure (BP) varying from 140 to $180 \mathrm{mmHg}$ systolic, and even higher in select patients. ${ }^{[4]}$

Endovascular therapy with cerebral angioplasty and/or administration of intra-arterial vasodilators are indicated in patients who fail medical treatment or where medical treatment is contraindicated (unsecured aneurysms or cardiac contraindications for hypertension) ${ }^{[4]}$ These endovascular procedures are usually performed with anaesthesia. Optimising the haemodynamic parameters to maintain cerebral perfusion is one of the key challenges during and after the procedure ${ }^{[5]}$ Increased hypertension may be needed to maintain perfusion through the vessels in spasm during the angioplasty. There is however an increase in risk of haemorrhage, vessel rupture or cerebral oedema during and after the angioplasty. Previous studies have discussed the haemodynamic management of patients undergoing intra-arterial vasodilator therapy for cerebral vasospasm. ${ }^{[6,7]}$ However, there is limited information on the anaesthetic care of patients undergoing endovascular treatment for cerebral vasospasm and perioperative haemodynamic management.

The objective of our study was to review the haemodynamic management and anaesthetic care of patients undergoing endovascular therapy for cerebral vasospasm in our institution.

\section{MATERIALS AND METHODS}

\section{Subjects}

After approval by the institutional review board, we conducted a retrospective cohort study of all adult patients, who underwent endovascular therapy for cerebral vasospasm secondary toSAH at our institution. Patients who were 18 years of age or older and had received endovascular therapy for vasospasm between April 2006 and September 2012, at Toronto Western Hospital, were included. We excluded patients who had confirmed vasospasm on angiogram but did not undergo endovascular treatment such as angioplasty, stenting or intra-arterial injection of a vasodilator agent and patients who had vasospasm after non-aneurysmal SAH such as arteriovenous malformations or intra ventricular haemorrhage. Our institutional practice in the management of patients with SAH followed the guidelines established by the American Heart Association. ${ }^{[5]}$
Patients received external ventricular drains for intracranial pressure monitoring and hydrocephalus as indicated. Aneurysms were secured early with endovascular coiling or surgical clipping. After this, management of BP was to maintain mean arterial pressures (MAP) of 90 to $100 \mathrm{mmHg}$ and central venous pressures (CVP) of 10-12 $\mathrm{mmHg}$. Gastric ulcer and thromboembolism prophylaxis were given as appropriate. Normothermia and euglycaemia were maintained. Surveillance for vasospasm was done with both daily clinical examinations and transcranial Doppler (TCD) measurements. Clinical vasospasm was suspected when there was a neurocognitive deficit and/or a change in blood velocity most commonly in the middle cerebral artery with TCD monitoring. Radiological diagnosis of vasospasm was considered when there was a narrowing of the cerebral arteries as shown with of either one of the modalities: Computed tomography angiography (CTA), magnetic resonance angiography (MRA), and/or catheter angiography. A multidisciplinary team involving neurosurgeons, neuroradiologists and neurointensivists were involved in the care of these patients. Patients with clinical vasospasm were treated initially with haemodynamic augmentation with infusion of vasopressors to maintain a systolic $\mathrm{BP}$ (sBP) of 140 to $170 \mathrm{mmHg}$, and fluid loading. Endovascular treatment was considered, if there was no clinical improvement or patient developed worsening of neurological symptoms despite BP augmentation after 12 hours. All patients were cared for in the neurosurgical intensive care unit (ICU) or in a high dependency neurosurgical unit.

The electronic patient record was reviewed for all identified patients. Data extracted include patient demographics, co-morbidities, and details of SAH, treatment modalities, complications, hospital mortality and discharge destinations. Anaesthesia records were used to abstract intraoperative data including haemodynamic data, vasopressor use and anaesthetic agents. Data on the onset, location and severity of cerebral vasospasm, nature of endovascular treatment, complications and outcome were obtained from the radiologist procedure notes. Post procedure haemodynamic management and complication data were obtained from ICU flow charts.

\section{Haemodynamic data}

Haemodynamic data collected include patients' $\mathrm{B} P$ values recorded every 15 minutes, during the period of 12 hours pre-angioplasty to 24 hours post angioplasty. From this data, the high, low, and average (mean) BP (systolic and diastolic) values were calculated for pre and post-angioplasty periods. Neurosurgical team often used the systolic blood pressure (instead of MAP) parameters to set their target haemodynamic goals. Hence sBP values were used in the analysis. The differences in sBP pre angioplasty were compared with the post-angioplasty recordings. We also assessed the differences between the predetermined targeted and the actual recorded sBP 
post- endovascular treatment. The requirements for and the choice of inotropes used were analysed.

\section{Endovascular treatment}

The type of endovascular treatment that the patient received was solely decided by the interventional neuroradiologist performing the procedure based on location of the vasospasm and vessel calibre as seen on the cerebral angiogram. The severity of cerebral vasospasm was graded into mild ( $<40 \%$ narrowing of vessels), moderate (40-75\% vessel narrowing), severe $(>75 \%$ narrowing) vasospasm and complete obliteration of vessel.

\section{Anaesthetic management}

The anaesthetic management of all the patients in the neuroradiology suite for the endovascular treatment was at the discretion of individual anaesthesiologist, who managed the case. All patients had standard anaesthetic monitoring, which included electrocardiogram, invasive and non-invasive BP, pulse oximetry, end-tidal carbon dioxide and temperature. Typically, anaesthesia was induced with an intravenous bolus of propofol and opioid (fentanyl or remifentanil) followed with an intubating dose of rocuronium. Some patients arrived from ICU with an endotracheal tube already in situ. The maintenance of anaesthesia was with oxygen, air, <1.0 minimum alveolar concentration inhalational agent (desflurane or sevoflurane), opioid (fentanyl or remifentanil), and intermittent muscle relaxant (rocuronium) all at the discretion of the attending anaesthesiologist.

\section{Statistics}

Descriptive statistics were used to characterise baseline clinical characteristics. Discrete variables were expressed as absolute numbers and percentages. In normally distributed data, variables are shown as mean \pm standard deviation (SD). The differences in the pre and postangioplasty sBP were determined using paired $t$ tests.

\section{RESULTS}

During the 6- year study period, 235 adult patients presented with $\mathrm{SAH}$, of whom 51 patients met our inclusion criteria. Six patients were excluded for incomplete and/or missing records. We collected and reviewed the electronic medical and anaesthesia records of a total of 45 patients.

\section{Patient Characteristics}

Demographics of the patients are shown in Table 1. Forty-five patients had a total of 47 endovascular interventions with 2 patients having two procedures at separate times. The aneurysm was located in the anterior circulation in $66.7 \%$ patients. Coiling of the aneurysm was the treatment of choice in $60 \%$ of the patients. Onset of vasospasm was on average around day 7 after SAH (range 2-15 day) [Figure 1]. Prior to catheter angiography, CTA was used to confirm the

\section{Table 1: Patient characteristics and endovascular treatment}

\begin{tabular}{|c|c|}
\hline Number of patients $(n)$ & 45 \\
\hline Number of interventions $(n)$ & 47 \\
\hline Age, (yr) (mean $\pm S D$ (range) & $51.6 \pm 10.4(34-79)$ \\
\hline Gender: Female/male, $n(\%)$ & $33 / 12(73.3 / 36.7)$ \\
\hline ASA physical status grade: $3 / 4, n(\%)$ & $2 / 43(4.4 / 95.6)$ \\
\hline \multicolumn{2}{|l|}{ Location of aneurysm, $n(\%)$} \\
\hline Anterior circulation & $30(66.7)$ \\
\hline Posterior circulation & $11(24.4)$ \\
\hline Both & $4(8.9)$ \\
\hline \multicolumn{2}{|l|}{ Treatment of aneurysm, $n(\%)$} \\
\hline Coiling & $27(60)$ \\
\hline Clipping & $18(40)$ \\
\hline \multicolumn{2}{|l|}{ Vasospasm severity, $n(\%)$} \\
\hline Mild & $5(10.6)$ \\
\hline Moderate & $14(29.8)$ \\
\hline Severe & $16(34.1)$ \\
\hline Complete narrowing & $12(25.5)$ \\
\hline \multicolumn{2}{|l|}{ Vasospasm location, $n(\%)$} \\
\hline Anterior circulation & $33(73.3)$ \\
\hline Posterior circulation & $3(6.7)$ \\
\hline Both & $9(20)$ \\
\hline \multicolumn{2}{|l|}{ Treatment of vasospasm, $n(\%)$} \\
\hline Angioplasty & $47(100)$ \\
\hline Endovascular stent & $1(2.1)$ \\
\hline Intra-arterial vasodilator therapy & $16(34)$ \\
\hline \multicolumn{2}{|l|}{ Intra-arterial vasodilator therapy, $n(\%)$} \\
\hline Nimodipine & $12(75)$ \\
\hline Verapamil & $2(12.5)$ \\
\hline Milrinone & $2(12.5)$ \\
\hline
\end{tabular}

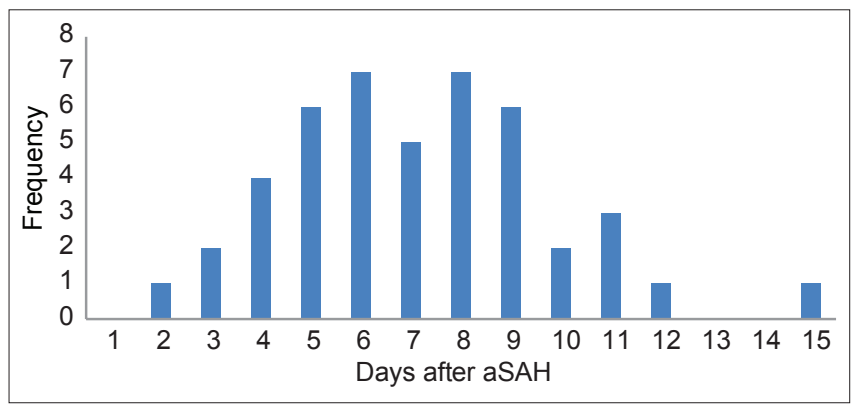

Figure 1: Distribution of days after initial SAH on which patients underwent endovascular therapy for treatment of vasospasm $(n=45)$ 
vasospasm in 39 patients. Vasospasm was usually seen in multiple vessels in the same patient regardless of the site of ruptured aneurysm and was present unilaterally in $80 \%$ of the patients. Moderate to severe vasospasm occurred in $63 \%$ of patients.

\section{Endovascular treatment and anaesthesia management}

The endovascular modality used for treatment of the vasospasm is outlined in Table 1 . All patients underwent balloon angioplasty, which was done mainly for proximal vessel spasm. Majority of the patients $(40 / 45)$ had angioplasty of 2 or more vessels while 5 patients had angioplasty of single proximal vessel. One patient had angioplasty of different vessels on two separate occasions. In addition, 15 patients had an intra-arterial injection of a vasodilator agent. Nimodipine (1-3mg), Verapamil $(5 \mathrm{mg})$ and Milrinone (5-8 mg) were commonly used agents for both proximal and distal vessel spasms. One patient had 2 intra-arterial treatments, each on separate occasions. Improved filling in the vessels was considered as a measure of successful treatment of vasospasm and angioplasty was successful in reversing the proximal vessel vasospasm in all patients.

All patients had a general anaesthesia for the procedure, and $19(40.4 \%)$ patients came from ICU already sedated, intubated and ventilated. At the end of the procedure, $16(34 \%)$ patients were extubated awake immediately in the neuroradiology suite, the other $(66 \%)$ patients remained sedated and were transferred to ICU. All the patients who were initially from ICU were all transferred back to ICU intubated.

\section{Haemodynamic management}

The BP measurements are summarised in Table 2 and Figure 2. There was a significant difference between the pre-angioplasty and post-angioplasty average sBP. Pre-endovascular treatment, the average, minimum and maximum sBP (mean + SD) were $163+24,137+27$ and $178+20 \mathrm{mmHg}$ respectively. Post-endovascular treatment, the average, minimum and maximum sBP (mean + SD) were $178+18,152+23$ and $192+15 \mathrm{mmHg}$. Prior to endovascular treatment, $31(68.9 \%)$ patients required vasopressors to achieve their BP targets [Table 2]. Norepinephrine was the most commonly used. Vasopressin was needed in $5(11.1 \%)$ patients in addition to norepinephrine. In the post-endovascular period, $93.3 \%$ of patients needed vasopressor support for a mean duration of 7.5 days (range 1-20). All patients who had required vasopressors prior to endovascular treatment continued to need them. All patients were able to reach and maintain the haemodynamic targets that had been set by neurosurgical team post- endovascular therapy. However, pre-endovascular therapy, there was one patient whose actual average sBP did not achieve the targeted sBP.

\section{Outcome and complications}

Angioplasty was successful in reversing the cerebral vasospasm in all patients as assessed by both angiography and clinical signs [Table 3]. There were no intra-procedure complications. There was no worsening of renal function in patients as a result of contrast dye. Overall $36(80 \%)$ were discharged from hospital and 9 patients (20\%) died from various causes [Table 3]. Among the non-neurological causes of mortality, pneumonia and sepsis were the cause of death in two patients and congestive heart failure in another two patients. No other patients suffered major end-organ dysfunction (bowel ischaemia, renal failure etc) as a result of vasopressor use.

\section{DISCUSSION}

Endovascular therapy is an effective treatment for cerebral vasospasm and is gaining popularity in many centers, as there is some evidence that the neurological

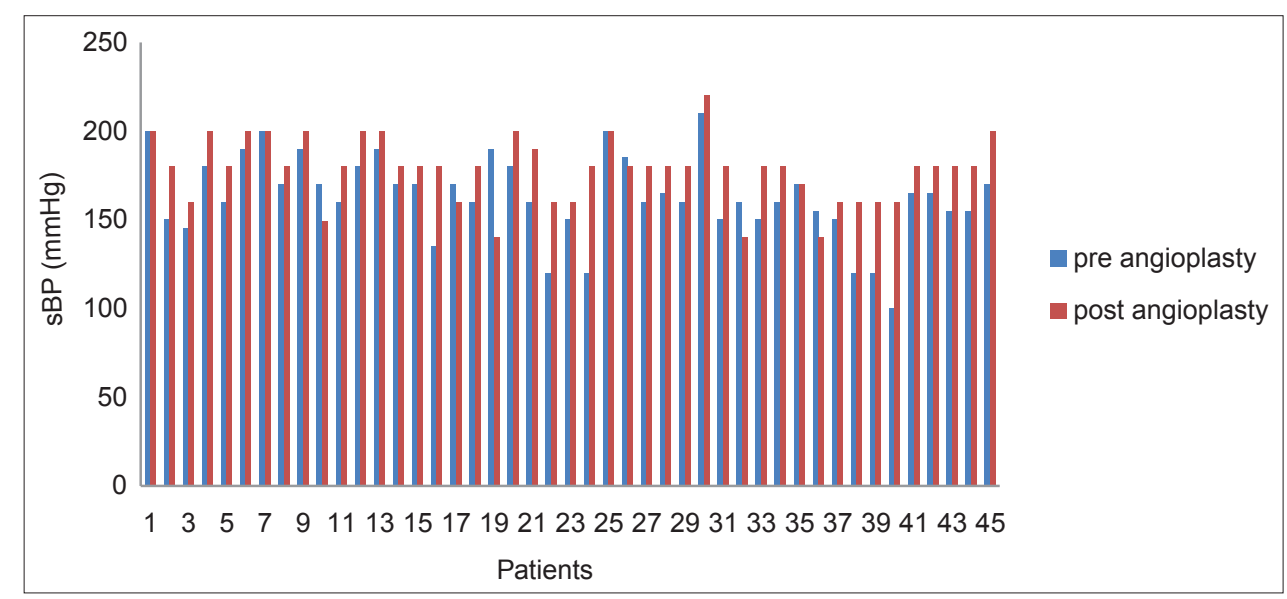

Figure 2: Systolic blood pressure pre and post endovascular therapy 
Table 2: Haemodynamic results

\begin{tabular}{llc}
\hline $\begin{array}{l}\text { Systolic blood pressure } \\
(\mathbf{m m H g}), \text { mean } \pm \text { SD }\end{array}$ & $\begin{array}{l}\text { Pre vs post } \\
\text { angioplasty }\end{array}$ & $\begin{array}{c}\mathbf{1 6 3} \mathbf{2 4} \mathbf{~ v s} \\
\mathbf{1 7 8} \mathbf{1 1 8}\end{array}$ \\
\hline Inotropes requirements, & None & $14(31.1)$ \\
$n(\%)$ & 1 type & $22(48.9)$ \\
& 2 types & $7(15.6)$ \\
& 3 types & $2(4.4)$ \\
& Pre vs post & $31(68.9) \mathrm{vs}$ \\
Types of inotropes, & angioplasty & $42(93.3)$ \\
$n(\%)$ & Norepinephrine & $28(62.2)$ \\
& Phenylephrine & $7(15.6)$ \\
& Vasopressin & $5(11.1)$ \\
& Dopamine & $2(4.4)$ \\
\hline
\end{tabular}

$n=$ Number of patients. Data expressed as mean $+/-$ SD (range) or as number (\%). ${ }^{*} P=0.000007$ ( $t$ test), significant difference between the systolic blood pressures pre- and post angioplasty

\section{Table 3: Outcomes}

\begin{tabular}{lc}
\hline Disposition at discharge, $n(\%)$ & $10(22.2)$ \\
Home & $26(57.8)$ \\
Rehabilitation & \\
Death, $n(\%)$ & $5(11.2)$ \\
Brain stem death & $2(4.4)$ \\
Cardiac arrest & $2(4.4)$ \\
Septic shock
\end{tabular}

$n=$ Number of patients. Data are reported as number (\%)

outcome of patients may be better with early intervention when compared to late endovascular therapy. ${ }^{[8-11]}$ In our institution, endovascular treatment is routinely considered, if there are no clinical improvements or there is worsening of neurological symptoms after 12 hours of BP augmentation. Endovascular therapy has been shown to be a safe procedure with minimal complications and a high success rate. The angiographic and clinical success rates of endovascular treatment of vasospasm have been quoted to be 98$100 \%$ and $65-70 \%$, respectively. ${ }^{[8-10]}$ Complications have been reported at a rate of $3.2 \%$ in one study of 189 patients undergoing endovascular treatment of vasospasm. ${ }^{[12]}$ The complications reported included vessel rupture (1), arterial dissections (3), severe increase in intracranial pressure (1) and thromboembolism (1). Different endovascular techniques are often used in the treatment of vasospasm. Balloon angioplasty alone is often effective in the treatment of vasospasm. Various intra-arterial vasodilator therapy such as milrinone, verapamil and nimodipine are also used. Nimodipine has a long half-life of 9 hours and was the most commonly used agent in our institution. Papaverine is the least used agent because of its short half-life and possible neurotoxic effect. ${ }^{[13]}$ Currently, there is little information to help decide on which vasodilator is superior, thus the choice of vasodilator varies with the preferences of the individual institution and radiologist.

Cerebral vasospasm will often affect multiple vessels in the same patient involving both proximal and distal vessels regardless of the location of initial bleed or aneurysm. Severity of vasospasm varies from mild narrowing to complete occlusion. In our data, $63 \%$ of patients had moderate to severe narrowing and $25 \%$ had complete occlusion. The timing of vasospasm after SAH in our study was around 7 days, which corresponds with previously reported figures. ${ }^{[2]}$ Even though clinical and radiographic vasospasms are associated, their incidence and presentations are different. Only $50 \%$ of patients with radiographic vasospasm present with ischaemic neurological symptoms (clinical vasospasm).${ }^{[14]}$ Similarly, the severity of clinical neurological symptoms does not correlate to severity of arterial spasm seen on cerebral angiogram. ${ }^{[14]}$ The reasons for this discrepancy may be multifactorial and includes poor collateral circulation, genetic differences and physiological variations in cellular ischaemic tolerance. ${ }^{[15]}$

The anaesthesia technique of choice in our institution was general anaesthesia as opposed to conscious sedation. These patients are challenging, as many of them are critically ill and on high dose of vasopressors. Transporting these patients between ICU and neuroradiology suite may also be challenging. In our study, $40 \%$ of the patients were intubated and ventilated in ICU prior to angioplasty due to altered state of consciousness. In addition, $66 \%$ of the patients remained sedated, intubated, mechanically ventilated when transferred back to ICU. Under general anaesthesia haemodynamic goals may be difficult to achieve, as anaesthetic agents may result in hypotension as there is no surgical stimulation. However, general anaesthesia provides the anaesthesiologists with airway control in the event of complications such as a re-bleed or arterial dissections. Also, the anaesthesiologist is able to control ventilation and $\mathrm{PaCO} 2$ as well as providing a motionless field with short apneic periods to facilitate mapping of the cerebral vasculature and advancement of micro catheters.

Our study showed that $68.9 \%$ of patients with clinical vasospasm prior to endovascular treatment required support of their BP with vasopressors to maintain a predetermined systolic blood pressure of 140 to $170 \mathrm{mmHg}$. After endovascular treatment, even more patients $(93.3 \%)$ required vasopressor support to maintain a higher BP. The reasons for this increased need for vasopressors after endovascular therapy are not completely understood. Firstly, this may be related to the fact that these patients are already developing increased severity of their clinical vasospasm. Also, cerebral vasospasm often occurs in multiple vessels both proximal and distal. Angioplasty is usually successful 
in treating vasospasm of the proximal vessels, but patients may still have vasospasm in many small distal vessels. Thus augmentation of the blood pressure was required for this. Secondly, this could be due to the use of intra-arterial vasodilator. Other studies have shown increased requirements of norepinephrine (50\%) and phenylephrine $(60 \%)$ after the use of intrarterial vasodilators ${ }^{[6]}$ However, only $16(34 \%)$ patients of our cohort had intra-arterial vasodilator injected.

The incidence of significant end organ damage was very low in our study in spite of the high percentage of patients needing vasopressors. High doses of vasopressors can lead to end organ damage such as bowel ischaemia, renal failure and myocardial ischaemia. ${ }^{[7]}$ In our study, two patients suffered cardiac morbidity and mortality; however, both patients also had prior history of cardiac ischaemia with poor left ventricle systolic function, which might have contributed to this. A possible reason for low incidence of end organ injury in our patients may be due to the presence of single organ injury (brain) unlike many other critical care patients with multi-organ failures from hypo-perfusion secondary to heart failure or sepsis.

\section{Limitations}

Our study has many limitations. The most important is the retrospective design and the reliance on medical records not designed for data collection specific to this study. It was also done in a single centre with a relatively small number of patients. Consequently, there are constraints on our conclusions. As this study was a non-randomised and non-prospective in patient selection, treatment bias is one of the major issues. As a result we cannot conclude what blood pressure targets are best for these patients.

\section{CONCLUSION}

Cerebral vasospasm affects multiple vessels in the same patient. Despite endovascular therapy being a successful intervention for proximal vessel spasm, most patients still required induced hypertension and even higher levels post angioplasty compared to pre angioplasty. There was no significant end-organ damage from the use of vasopressors. There is a need for more prospective studies to validate these findings and to determine the rationale of haemodynamic management.

\section{REFERENCES}

1. Rivero-Arias O, Gray A, Wolstenholme J. Burden of disease and costs of aneurysmal subarachnoid haemorrhage (aSAH) in the United Kingdom. Cost Eff Resour Alloc 2010;8:6.

2. Harrod CG, Bendok BR, Batjier HH. Prediction of cerebral vasospasm in patients presenting with aneurysmal subarachnoid hemorrhage: A review. Neurosurgery 2005;56:633-54.

3. Kassell NF, Peerless SJ, Durward QJ, Beck DW, Drake CG, Adams HP. Treatment of ischemic deficits from vasospasm with intravascular volume expansion and induced arterial hypertension. Neurosurgery 1982;11:337-43.

4. Connolly ES Jr, Rabinstein AA, Carhuapoma JR, Derdeyn CP, Dion J, Higashida RT, et al. Guidelines for the management of aneurysmal subarachnoid hemorrhage: A guideline for healthcare professionals from the American Heart Association/ American Stroke Association. Stoke 2012;43:1711-37.

5. Bederson JB, Connolly ES Jr, Batjer HH, Dacey RG, Dion JE, Diringer MN, et al. Guidelines for the management of aneurysmal subarachnoid hemorrhage. A statement for healthcare professionals from a special writing group of the Stroke council, American Heart Association. Stroke 2009;40:994-1025.

6. Schmidt U, Bittner E, Pivi S, Marota JJ. Hemodynamic management and outcome of patients treated for cerebral vasospasm with intraarterial nicardipine and/or milrinone. Anesth Analg 2010;110:895-902.

7. Treggiari MM. Participants in the International Multi-disciplinary Consensus Conference on the Critical Care Management of Subarachnoid Hemorrhage. Hemodynamic management of subarachnoid hemorrhage. Neurocritical Care 2011;15:329-35.

8. Brothers MF, Holgate RC. Intracranial angioplasty for treatment of vasospasm after subarachnoid hemorrhage: Technique and modifications to improve branch access. AJNR Am J Neuroradiol 1990;11:239-247.

9. Higashida RT, Halbach VV, Dowd CF, Dormandy B, Bell J, Hieshima GB. Intravascular balloon dilatation therapy for intracranial arterial vasospasm: Patient selection, technique and clinical results. Neurosurg Rev 1992;15:89-95.

10. Eskridge JM, Newell DW, Winn HR. Endovascular treatment of vasospasm. Neurosurg Clin N Am 1994;5:437-47.

11. Rosenwasser RH, Armonda RA, Thomas JE, Benitez RP, Gannon PM, Harrop J. Therapeutic modalities for the management of cerebral vasospasm: Timing of endovascular options. Neurosurgery 1999;44:975-80.

12. Jun P, Ko NU, English JD, Dowd CF, Halbach VV, Higashida RT, et al. Endovascular treatment of medically refractory cerebral vasospasm following aneurysmal subarachnoid hemorrhage. AJNR Am J Neuroradiol 2010;31:1911-6.

13. Smith WS, Dowd CF, Johnston SC, Ko NU, DeArmond SJ, Dillon WP. et al. Neurotoxicity of intra-arterial papaverine preserved with chlorobutanol used for the treatment of cerebral vasospasm after aneurysmal subarachnoid hemorrhage. Stoke 2004;35:2518-22.

14. Vergouwen MD, Vermeulen $M$, van Gijn J, Rinkel GJ, Wijdicks EF, Muizelaar JP, et al. Definition of delayed cerebral ischemia after aneurysmal subarachnoid hemorrhage as an outcome event in clinical trials and observational studies: Proposal of a multidisciplinary research group. Stroke 2010;41:2391-5.

15. Yundt KD, Grubb RLJr, Diringer MN, Powers WJ. Autoregulatory vasodilation of parenchymal vessels is impaired during cerebral vasospasm. J Cereb Blood Flow Metab 1998;18:419-24.

How to cite this article: Tan A, Tharmaradinam S, Mariappan R, Manninen P, Venkatraghavan L. Haemodynamic and anaesthetic management of patients undergoing endovascular therapy for cerebral vasospasm. J Neuroanaesthesiol Crit Care 2014;1:204-9.

Source of Support: Nil, Conflict of Interest: None declared. 